\title{
La formación en derechos humanos y desarrollo sostenible como educación liberadora
}

Fecha de recepción: 21 de febrero de 2020.

Fecha de aceptación: 27 de abril de 2020.

\begin{abstract}
Resumen
Paulo Freire desarrolló a lo largo de su obra conceptos como emancipación, pedagogía crítica, empoderamiento, construcción mutua y colectiva del conocimiento, cambio social y transformación política. Estas ideas se corresponden con los objetivos que persigue la educación en derechos humanos. Asimismo, en la actualidad, para lograr una verdadera educación liberadora se hace necesario incorporar la educación para el desarrollo sostenible. A propósito del centenario del nacimiento del educador brasileño, este ensayo de reflexión teórica pretende conectar algunas de sus ideas principales con las tendencias actuales en materia de educación en derechos humanos y desarrollo sostenible. Finalmente, se concluirá en la necesidad, importancia y potencial de integrar este tipo de educación efectivamente en la formación de todo individuo, a través de todos los canales posibles. A fin de cuentas, se trata de un recurso fundamental para garantizar la dignidad de las personas. Solo así se podrá sostener e impulsar el legado de Freire.
\end{abstract}

Palabras clave: educación liberadora, educación en derechos humanos, educación en desarrollo sostenible

\section{Education in human rights and sustainable development as a liberating education}

\begin{abstract}
Paulo Freire's work includes concepts like emancipation, critical pedagogy, empowerment, collective construction of knowledge, social change and transformation. These ideas are clearly linked with the objectives pursued by education in human rights. Furthermore, in order to achieve a true liberating education these days we need to include an education for sustainable development. Considering the 100th anniversary of Freire's birth, this essay intends to connect some of his main ideas with the current
\end{abstract}


trends on education in human rights and sustainable development. It will also reflect on the necessity, importance and potential of integrating this education into the upbringing of every person, through every channel available. In the end, it is a fundamental tool to guarantee the dignity of people and to sustain and promote Freire's legacy.

Keywords: liberating education, education in human rights, education for sustainable development

\section{Introducción}

Paulo Freire desarrolló a lo largo de su obra conceptos como educación liberadora, pedagogía crítica, emancipación, empoderamiento, construcción mutua y colectiva del conocimiento, cambio social y transformación política. El pedagogo brasileño consideraba fundamental el respeto a la autonomía y a los saberes del educando, afirmaba que docentes y estudiantes se educan mutuamente, cuestionaba el método bancario y destacaba el potencial de la educación para modificar las injusticias del mundo (Freire, 1999a, 1999b, 2002). También promovía la tolerancia y la no discriminación y luchó para que las personas más necesitadas - los oprimidostuvieran su propia voz y cambiaran su realidad, entre otras propuestas (Freire, 1999a, 2002, 2012).

Todas estas ideas están sumamente ligadas a los objetivos que persigue la educación en derechos humanos. Asimismo, en los últimos años ha cobrado cada vez más fuerza la concientización sobre el cuidado del ambiente y la necesidad de luchar contra el cambio climático. En este sentido, también se ha consolidado la postura que remarca la interdependencia entre derechos humanos, desarrollo sostenible y la protección del planeta (Corte IDH, 2017, 2020, 2019). Quizás uno de los hitos más relevantes sobre este punto se haya producido en 2015 con la adopción desde Naciones Unidas de la Agenda 2030 que, con sus 17 Objetivos de Desarrollo Sostenible, abarca de manera integral los principales desafíos de los próximos años.

Con motivo del centenario del nacimiento de Paulo Freire, este ensayo de reflexión teórica intenta, por un lado, conectar algunas de las principales ideas del educador con los propósitos específicos actuales de la educación en derechos humanos. Es decir, tomar a Freire como un marco o como una influencia para la educación en derechos humanos. Para ello, se comenzará analizando la definición de educación en derechos humanos, se profundizará el concepto con las opiniones de la doctrina especializada y se irán vinculando los objetivos, desde un enfoque de derechos. Por otro lado, el segundo propósito del artículo es incluir también en este lazo a la educación por el desarrollo sostenible, un concepto imposible de separar de este marco si tenemos la intención de ofrecer una verdadera educación liberadora y transformadora. Por último, como la realidad política muestra pocos avances en estos aspectos, insistir con la difusión de estos puntos a través de todos los canales posibles y promover el activismo aparece como una tercera contribución.

\section{Qué entendemos por educación en derechos humanos}

En 2011, la Asamblea General de las Naciones Unidas adoptó una de las definiciones de educación en derechos humanos más aceptadas en la actualidad (Tibbitts, 2017). En su Declaración de las Naciones Unidas sobre educación y formación en materia de derechos humanos indicó: 
1. La educación y la formación en materia de derechos humanos están integradas por el conjunto de actividades educativas y de formación, información, sensibilización y aprendizaje que tienen por objeto promover el respeto universal y efectivo de todos los derechos humanos y las libertades fundamentales, contribuyendo así, entre otras cosas, a la prevención de los abusos y violaciones de los derechos humanos al proporcionar a las personas conocimientos, capacidades y comprensión y desarrollar sus actitudes y comportamientos para que puedan contribuir a la creación y promoción de una cultura universal de derechos humanos.

2. La educación y la formación en materia de derechos humanos engloban: a) La educación sobre los derechos humanos, que incluye facilitar el conocimiento y la comprensión de las normas y principios de derechos humanos, los valores que los sostienen y los mecanismos que los protegen; b) La educación por medio de los derechos humanos, que incluye aprender y enseñar respetando los derechos de los educadores y los educandos; c) La educación para los derechos humanos, que incluye facultar a las personas para que disfruten de sus derechos y los ejerzan, y respeten y defiendan los de los demás (Asamblea General de las Naciones Unidas, 2011. Las cursivas son propias).

De esta definición se pueden destacar algunos elementos centrales, como el objeto de promover el respeto de los derechos humanos, prevenir abusos y vulneraciones e impulsar una cultura de derechos humanos. Asimismo, la Declaración menciona el concepto de educación sobre derechos (aspecto temático, de contenido), por medio de o a través de los derechos humanos (enseñar con el ejemplo, coherencia), y para los derechos humanos (propósito transformador, de incidencia, influencia). Es fundamental que estas tres dimensiones de la educación en derechos humanos estén presentes siempre.

Además de la educación en derechos humanos, existen propuestas similares que, aunque llevan distintos nombres, pueden quedar incluidas en este concepto. Por ejemplo, se habla de educación por la ciudadanía, educación ciudadana, educación cívica, educación para la paz, cultura para la paz, educación por la democracia, educación en género, educación ambiental, educación multicultural, formación en valores, educación ética, educación para la convivencia, la tolerancia y la no discriminación, etcétera. No se pretende en este artículo puntualizar en cada propuesta y diferenciarlas, sino al menos mencionar estos puntos que integran el marco teórico y que tienen muchos contenidos solapados. Para el presente artículo, llamaremos educación en derechos humanos a las actividades que tienen por objeto promover el respeto de los derechos humanos, contribuir a la prevención de los abusos y promocionar una cultura universal de derechos humanos, como se definió anteriormente. Probablemente, bajo este paraguas queden incluidos muchos aspectos de las propuestas similares.

Ahora bien, en ciertos ámbitos, parece necesario aclarar algunos puntos esenciales frente a una posible desconfianza que pudiera despertar la educación en derechos humanos. Por ejemplo, como se desarrollará a continuación, enseñar derechos humanos no es necesariamente bajar línea; la verdadera enseñanza en derechos humanos propone un pensamiento crítico propio, que es algo muy diferente a tratar de imponer un pensamiento particular. Y enseñar derechos humanos no es solamente tocar temas de dictaduras, guerras, gobiernos militares y memoria, verdad y justicia (que, por supuesto, deben ser una parte importante del contenido). Es necesario incluir temas de igualdad y no discriminación, educación sexual integral, derechos económicos, sociales, culturales y ambientales, etcétera.

Por otra parte, los derechos humanos y la educación en derechos humanos son "el referente ético más adecuado para las sociedades actuales" (Pérez Aguirre, 2007: 51) pero también representan obligaciones concretas para los Estados. Es decir, "no se trata de bellas ideas flotando en el espacio; son instrumentos legales basados en valores humanos sobre los que hemos acordado a nivel internacional, regional y 
nacional" (Instituto Danés de Derechos Humanos, 2013: 15). Algunos de los tratados internacionales que consagran el derecho de toda persona a recibir educación en derechos humanos y desarrollo sostenible son el Pacto Internacional de Derechos Económicos, Sociales y Culturales, la Convención sobre los Derechos del Niño y el Protocolo de San Salvador.

Además, varios organismos de Naciones Unidas se han expresado sobre la importancia de esta clase de formación. Por ejemplo, el Comité para la Eliminación de la Discriminación contra la Mujer (2017: 1) señaló que "la educación cumple una función esencial, transformadora y de empoderamiento en la promoción de los valores de los derechos humanos y se considera la vía para la igualdad de género y el empoderamiento de las mujeres". La Relatora Especial sobre el derecho a la educación (2018) consideró que puede proteger a los niños de la violencia, la explotación, las actividades delictivas y las enfermedades, y que tiene el potencial de promover la paz y enseñar tolerancia. Por último, el Comité de Derechos del Niño (2016) sostuvo que la escuela secundaria debería facilitar la participación activa de los adolescentes, desarrollar el respeto por los derechos humanos y las libertades fundamentales, promover el compromiso cívico y preparar a la juventud para llevar una vida responsable en una sociedad libre.

Ahora bien, también es cierto, y no se desconoce, que existen críticas generales hacia el movimiento, el lenguaje o el discurso de los derechos humanos. A modo de ejemplo, Mónica Fernández (2019) cuestiona que la enseñanza y el aprendizaje de los derechos humanos son parte de saberes hegemónicos y de una estrategia colonizadora que tiende a la dominación. La autora reclama que los derechos reconocidos no se practican en la realidad, que se impide el diálogo intercultural y que se trata de "una nueva máscara del poder" (Fernández, 2019: 141).

Sin pretender agotar una extensa discusión que excede el presente artículo, sí se podrían destacar al menos algunos puntos. En primer lugar, la construcción del régimen del derecho internacional de los derechos humanos luego de la Segunda Guerra Mundial en la Organización de las Naciones Unidas contó con una colaboración fundamental de países del Hemisferio Sur (Sikkink, 2017). Más aún, específicamente en el caso de América Latina, cabe mencionar que la Declaración Americana de Derechos y Deberes del Hombre preexistió a la Declaración Universal de Derechos Humanos, y que el Sistema Interamericano de Protección de Derechos Humanos, conformado principalmente por la Comisión Interamericana de Derechos Humanos y la Corte Interamericana de Derechos Humanos, cuenta con un desarrollo propio que es una referencia a nivel mundial.

En segundo lugar, más allá de que los propósitos enunciados en los instrumentos de derechos humanos sean completamente contrarios a lo que sugieren las críticas, la falta de su efectividad no puede ser atribuida exclusivamente al propio régimen. Es decir, el incumplimiento y las violaciones de los derechos humanos no son necesariamente responsabilidad del movimiento o del discurso sino que hay involucradas cuestiones sociales, económicas, culturales, políticas, etcétera. De hecho, el poco desarrollo que tiene la educación en derechos humanos quizás explique por qué la implementación de los tratados no es tan universal.

Por último, que los objetivos perseguidos parezcan utópicos tampoco debe servir para invalidarlos. El horizonte y la posibilidad de pensar y hacer un mundo más justo es fundamental y es un elemento que también se encuentra muy presente en la obra de Freire (1999a). Explica Kohan (2020: 148): "el principal enemigo político de Paulo Freire no es el capitalismo o cualquier sistema político específico, sino el fatalismo, la convicción de que 'las cosas no pueden ser de otra manera', la comprensión de la historia como determinismo y no como posibilidad". A esto se refiere Freire (2012) cuando habla 
de que no somos sino que estamos siendo y que el futuro no nos determina, sino que nosotros debemos luchar para hacer el futuro. Precisamente, la educación en derechos humanos y en desarrollo sostenible implica sembrar la semilla para que se piense que el mundo puede - y debe - ser de otra manera, que no es algo fijo o dado, brindando incluso herramientas jurídicas para que eso suceda. Desde ya, no lo alcanzará por sí sola y será necesario complementarla con otros métodos, pero definitivamente es un esfuerzo hacia la dirección correcta, aun con sus limitaciones.

En este sentido, para que se puedan cumplir los objetivos de la educación en derechos humanos y en desarrollo sostenible, sería ideal que se cumplieran las siguientes condiciones: por un lado, es clave que el contenido y el enfoque de los derechos humanos aparezcan de manera transversal en todas las materias y en todos los niveles - considerando las particularidades de cada grupo- y no como un curso o un taller particular aislado (IIDH, 2013; Ronconi, 2017; Medici, 2018). Por otro lado, es fundamental no quedarse solamente en el nivel teórico o académico. Es necesario bajar el contenido y conectarlo a la realidad de los estudiantes, hacerlos entender que los derechos humanos están presentes constantemente, lograr que puedan relacionar los temas con sus vidas personales y con las de sus amigos y sus familias, para poder comprender mejor estas cuestiones de justicia, respeto y solidaridad (Pérez Aguirre, 2007; Pinto, 2010). Como sostiene Ana María Rodino (2014: 259), "muchas problemáticas sociales no se analizan relacionadas con los derechos humanos, aunque sí lo están (por ejemplo, las migraciones y desplazamientos, la pobreza, la sostenibilidad del desarrollo, la salud sexual y reproductiva y la violencia comunitaria e intrafamiliar)".

\section{La educación en derechos humanos como educación liberadora}

Varios de los objetivos mencionados que persigue la educación en derechos humanos se solapan con los propósitos enunciados en los escritos de Freire. A esto hace referencia la "conexión" mencionada, a objetivos en común, puntos de contacto, a tomar la obra de Freire como influencia o antecedente de la educación en derechos humanos y desarrollo sostenible, a explicitar esta relación. $\mathrm{O}$, en otras palabras, a repensar la educación liberadora en términos actuales de educación en derechos humanos.

Para volver a la definición tripartita, cuando se habla de educación sobre derechos humanos, se está haciendo referencia a un primer paso necesario o a una forma embrionaria de empoderamiento, que consiste en conocer nuestros derechos, comprender las injusticias y descubrir los canales para revertirlas. Así, "la alfabetización puede facultar a hombres y mujeres para el ejercicio de los derechos humanos y puede funcionar como un instrumento para investigar las formas en que se configuran las definiciones culturales de género, raza, clase" (El Achkar, 2002: 111).

El conocimiento de las normas y las instituciones que protegen los derechos humanos no es algo meramente académico sino que otorga la capacidad y el poder de actuar, de intervenir en la promoción, protección y difusión de los derechos (Magendzo, 2001). Incluso, también puede ser una manera de empoderarse el descubrir, por un lado, que los propios valores, las opiniones individuales o la percepción personal de la realidad y del deber ser son consistentes y se identifican con estándares internacionales de derechos humanos; y, por otro lado, detectar que existen otras personas que también sufren vulneraciones o formas similares de discriminación (Tibbitts, 2017).

Por otra parte, cuando Freire reclama valorar los saberes y la autonomía de los educandos y afirma que enseñar exige saber escuchar, se hace presente la educación por medio de o a través de los derechos humanos. En términos del pedagogo brasileño, "el respeto 
a la autonomía y a la dignidad de cada uno es un imperativo ético y no un favor que podemos o no concedernos a los otros" (Freire, 1999b: 58). Más aún, Freire indica que "enseñar exige la corporificación de las palabras en el ejemplo" (Freire, 1999b: 35). Y estas ideas también se conectan con la opinión de que el conocimiento no se transmite de una persona a otra, sino que se construye mutuamente (Freire, 1999a).

Revalorizar al estudiante desde su capacidad y su conocimiento, tomarlo como sujeto de derecho y respetar sus derechos humanos es una manera de alejarse del modelo de educación bancaria, es una forma de sugerir que nadie educa a nadie, que nadie se educa a sí mismo y que las personas se educan entre sí (Freire, 1999a). Y aquí también aparece la necesidad de evitar el adoctrinamiento y de impulsar un verdadero pensamiento crítico. Así, el ideal es lograr un aula que sea "un espacio de acogida para nuestros estudiantes, sin que se sientan coaccionados a optar por una única interpretación del pasado reciente, sino abriendo espacios de diálogo genuino, transparente y respetuoso" (Franco Sentis et al., 2018: 229).

Por supuesto, esto no quiere decir que los estudiantes sepan todo o que estén en pie de igualdad con el docente. Claramente, quien está frente a una clase está a cargo de aportar conocimientos nuevos y de conducir el curso, pero debe comprender que también está aprendiendo, que sus oyentes pueden contribuir a su formación y a la de sus colegas, y que esta formación debe tender a la generación de un pensamiento individual y propio, que luego podrá coincidir o no con el suyo. Como señala Freire en su Pedagogía de la Esperanza, "cuanto más tolerantes, cuanto más transparentes, cuanto más críticos, cuanto más curiosos y humildes sean, tanto más auténticamente estarán asumiendo la práctica docente" (Freire, 2002: 77).

Ahora bien, probablemente el aspecto de la educación en derechos humanos más relacionado con las ideas de Paulo Freire sea el punto de educación para los derechos humanos. El objetivo de permitir que las personas ejerzan y disfruten de sus derechos y respetar y defender los derechos de los demás es claramente transformador, emancipador, una forma madura de empoderamiento. En este sentido, una verdadera educación en derechos humanos no implica solamente transmitir información, sino que significa adquirir los conocimientos, habilidades, valores y motivaciones necesarios para comprender y reivindicar los derechos (Tibbitts y Fernekes, 2011; IIDH, 2013).

Freire propuso a lo largo de su vida una educación que condujera hacia la liberación y la independencia. Luchó por promover una lectura crítica del mundo y por brindar herramientas no para adaptarse a los cambios, sino para generarlos y revertir las injusticias (Freire, 1999a, 2002, 2012). Precisamente, varios autores sostuvieron explícitamente que la educación en derechos humanos busca, primero, una transformación individual, donde se cambien los valores, las actitudes y conductas, para luego movilizar a las personas para que modifiquen y mejoren su entorno (Fisas, 1998; Magendzo, 2001; Pérez Aguirre, 2007; Tibbitts, 2018).

Asimismo, las metodologías y los recursos pedagógicos más apropiados para enseñar derechos humanos son aquellos que fomenten el espíritu crítico, que cuestionen el statu quo, que rechacen la dominación (Cussianovich, 2010; Tibbitts y Kirchschläger, 2010; Tibbitts, 2017). Acá también se ve esta conexión, relación o influencia entre Freire y la educación en derechos humanos. Se habla de "educar para la disidencia, la indignación, la desobediencia responsable, la elección con conocimiento y la crítica" (Fisas, 1998: 14). Y esto se logra a través de una educación problematizadora, con un enfoque controversial, que no le tenga miedo a la deliberación o al disenso, sino que trabaje y construya sobre eso (Siede, 2010; Magendzo y Pavez, 2018). Justamente, el diálogo es una herramienta pedagógica importantísima en Freire (1999a). 
Por ejemplo, se deben utilizar recursos como los juegos de rol, debates, o moot-courts (simulaciones de casos ante tribunales internacionales), que no solo promueven la tolerancia, el respeto y el reconocimiento del otro, sino que favorecen el aprendizaje porque les permite a los estudiantes interactuar con el objeto de estudio y ponerlo en práctica, en lugar de leer y escuchar sobre el tema solamente. De este modo, se conseguirá el efecto emancipador y transformador que buscaba Freire y que también busca actualmente la educación en derechos humanos. A fin de cuentas, la forma mediante la cual se enseñen los derechos humanos y quién los enseñe pueden influir marcadamente en la postura que tomen los estudiantes frente a ellos (Requesens Galnares, 2006).

En síntesis, se puede ver cómo la doctrina actual de la educación en derechos humanos se conecta con las propuestas de Freire, quien la influencia y le sirve como antecedente: hablamos de un conocimiento sobre la identidad, las normas, los valores y las formas de reclamar el cumplimiento de los derechos (educación sobre derechos humanos); con un aprendizaje centrado en el estudiante (educación por medio de o a través de los derechos humanos); y que promueva una reflexión crítica, que luego permita el empoderamiento, el activismo y la posibilidad de transformar la realidad de una manera compatible con los derechos humanos (educación para los derechos humanos) (Tibbitts, 2018).

Al mismo tiempo, la autocrítica que hace Freire en su Pedagogía de la Esperanza sobre el lenguaje "machista" de Pedagogía del Oprimido, por ejemplo, también se puede codificar en términos de derechos humanos. Sostiene el autor: "La discriminación de la mujer, expresada y efectuada por el discurso machista y encarnada en prácticas concretas, es una forma colonial de tratarla, incompatible por lo tanto con cualquier posición progresista" (Freire, 2002: 64). Y luego agrega: "El rechazo de la ideología machista, que implica necesariamente la recreación del lenguaje, es parte del sueño posible en favor del cambio del mundo" (Freire, 2002: 64).

Es decir, el pensamiento freireano también se ve influenciado por los derechos humanos, incluso expresamente. En su Pedagogía de la Indignación, el brasileño afirma que "la lucha a favor de los hambrientos (...) forma parte, en realidad, del dominio de la ética universal del ser humano tanto como la lucha a favor de los derechos humanos, dondequiera que se entable; del derecho a transitar libremente, del derecho a comer, a vestir, a pronunciar la palabra, a amar, a elegir, a estudiar, a trabajar; del derecho a creer y a no creer, del derecho a la seguridad y a la paz" (Freire, 2012: 167). Y continúa: "la gran tarea del poder político es garantizar las libertades, los derechos y los deberes, la justicia, y no respaldar la arbitrariedad de unos pocos contra la debilidad de las mayorías" (Freire, 2012: 169).

Por último, vale aclarar que la tendencia en general del derecho internacional de los derechos humanos incluye en este propósito de empoderamiento elementos de pluralismo y no discriminación. Por supuesto, ambos aspectos no se excluyen y es necesario buscar este gris entre la indignación y la tolerancia, entre la rabia y el amor, entre la ira y los sueños y la esperanza, en términos de Freire. Así como nuestros derechos se deben ejercer en un marco de respeto por los de otros, el objetivo final sería que la persona se empodere como agente de cambio dentro de un contexto de diversidad e inclusión. A esto debería apuntar el activismo, el cambio social y la mejora de la democracia. A fin de cuentas, se trata de revalidar el concepto de conjunto o comunidad, y comprender que uno es parte de un todo, que si el otro está mejor, yo también estaré mejor; básicamente, no dejar a nadie atrás, como pide la Agenda 2030.

De esta forma, a través de una efectiva educación en derechos humanos, las personas no solamente se deberían volver más comprensivas y tolerantes sobre sus diferencias, sino que también podrán contar con elementos para buscar una transformación política y exigir el cumplimiento de derechos mediante la presión desde abajo. Es decir, no 
solo se deberían prevenir abusos, hostigamientos y violaciones de derechos entre las personas, sino que también debería servir para concientizar sobre las obligaciones de los Estados a la hora de respetar y garantizar los derechos y, en consecuencia, ejecutar ese reclamo. En otras palabras, este tipo de conocimiento favorece que las personas puedan exigir la vigencia de los derechos humanos y que estén en permanente vigilancia frente a posibles vulneraciones (Magendzo, 2001).

\section{La necesidad de incorporar la educación para el desarrollo sostenible}

En los últimos años, la lucha por la conservación del ambiente fue adquiriendo cada vez más fuerza. Incluso generó movimientos políticos juveniles con considerable éxito en cuanto a su organización y capacidad de ser oídos por el poder público. Si bien el concepto de desarrollo sostenible nació a fines de la década de 1980, últimamente fue aumentando la concientización de la sociedad en general y la protección del planeta está muy presente en la agenda de hoy. No obstante, es necesario reconocer que el campo ambiental y del desarrollo sostenible es un área con menor trayectoria temporal y más nueva que la de los derechos humanos, y tal vez más pequeña y concreta. Aún así, se aclara que el presente apartado constituye un resumen que no agota la temática.

A modo de ejemplo, recientemente, la Corte Interamericana de Derechos Humanos (2017: 52; 2020: 85) afirmó que "existe un amplio reconocimiento en el derecho internacional sobre la relación interdependiente entre la protección al medio ambiente, el desarrollo sostenible y los derechos humanos". La Comisión Interamericana de Derechos Humanos (2019) también ha insistido con la necesidad de reconocer el carácter universal, indivisible, interdependiente e interrelacionado de los derechos humanos y de cerrar las brechas entre la protección de los derechos económicos, sociales, culturales y ambientales en comparación con los derechos civiles y políticos. Específicamente, sostuvo que "la relación estrecha entre los derechos humanos, el desarrollo sostenible y el medio ambiente abarca innumerables facetas y alcances" (CIDH, 2019: 35). Efectivamente, el cuidado de los recursos naturales y un modelo de desarrollo sostenible inciden de manera transversal sobre los derechos humanos y todos los contenidos que se enseñan en la escuela. Por eso, "el imperativo de desarrollo sostenible y estilos de vida sostenibles también debería ser un principio rector para la educación en derechos humanos" (Cho, 2018_37).

Retornando a Freire, el pedagogo brasileño pretendía de cierta forma trabajar para que en el futuro se pudieran hacer cosas que hasta el momento no eran posibles. La idea de desarrollo sostenible vendría a ser la otra cara de la misma moneda: cuidar las cosas hoy, para que sea posible disfrutarlas mañana. Básicamente, lo que busca este concepto es que las generaciones presentes aprovechen los recursos naturales para satisfacer sus necesidades pero sin comprometer el goce de las generaciones futuras. Esto también está relacionado con el principio de equidad intergeneracional. En este ámbito, también es fundamental la capacidad de poder adaptarse a una realidad cambiante, pero con el potencial de modificarla y mejorarla.

Justamente, como define Unesco, "la educación en desarrollo sostenible empodera a los educandos para tomar decisiones informadas y adoptar acciones responsables en favor de la integridad del ambiente, la viabilidad de la economía y una sociedad más justa, para las generaciones actuales y futuras, respetando la diversidad cultural" (Unesco, 2016: 25). Además, agrega que la educación en desarrollo sostenible "es una parte integral de una educación de calidad y transformativa" (Unesco, 2016: 25). En este sentido, es necesario incluir en la enseñanza aspectos de cambio climático, biodiversidad, consumo responsable y sustentable, entre otros contenidos. El objetivo primordial sería facilitar una transición hacía economías y sociedades más verdes, 
con educandos equipados con habilidades para trabajar en rubros ambientalmente amigables y motivados para adoptar modelos de vida más sustentables.

Ahora bien, al igual que sucede con el discurso de los derechos humanos, la idea de desarrollo y el concepto de desarrollo sostenible también han sido objeto de críticas. Nuevamente, queda por fuera del objeto de este ensayo realizar un análisis profundo sobre estos puntos, pero al menos se expondrán las principales ideas. En cierta forma, los cuestionamientos son similares y apuntan mayoritariamente a que el desarrollo sostenible es una nueva forma de dominación que impone el Norte hacia el Sur, que es una herramienta discursiva y que es inaplicable e inefectivo. A modo de ejemplo, se habla de "la amenaza de convertir la conciencia en retórica", es decir "convertir la conciencia de clase en corporativismo, o la solidaridad internacional y la lucha contra la pobreza en eslóganes publicitarios" (Abril, 2003: 76). Pero la solución sugerida vuelve a estar en la educación: "esto solo se combate reflexionando sobre cada una de nuestras acciones cotidianas, actuando localmente en casa, en la calle, en el aula... pero pensando globalmente, con sentido planetario" (ibídem).

Tampoco corresponde esquivar la falta de resultados masivos o revolucionarios o, como sugieren algunos autores, las "paradojas del presente: a más lucha contra la pobreza, más pobreza; a más cooperación para el desarrollo, menos desarrollo; a más desarrollo humano, menos humanos" (Capalbo et al., 2011: 9), pero el punto es, una vez más, si la responsabilidad es del concepto, de la idea, del objetivo o si, en realidad, hay otros factores más relevantes que, justamente, podrían intentar corregirse a través de una mejor educación y difusión de estas metas. Muchas veces se equipara el concepto de desarrollo con crecimiento o desarrollo económico cuando, justamente, la idea detrás del desarrollo sostenible es incluir necesariamente en la ecuación los aspectos sociales y ambientales. En términos de la Comisión Interamericana de Derechos Humanos, para que el desarrollo sea sostenible "se debe poner necesariamente el centro de atención en el bienestar y derechos de las personas y comunidades más que en las estadísticas económicas y mercancías" (CIDH, 2019: 34). En este sentido, es imperioso despegarse del materialismo y de la grosera acumulación de bienes, dinero y artefactos en desmedro del ambiente bajo la falsa premisa de que esto nos hará más felices. Es difícil negar que "la obsesión por el crecimiento económico nos ha llevado a traspasar los umbrales que la Tierra puede soportar" (Capalbo et al., 2011: 57). Entonces, siempre es válido recordar que crecimiento económico no es lo mismo que desarrollo.

El propio Freire, en su Pedagogía del Oprimido, también advertía sobre estas cuestiones: "a fin de comprobar si una sociedad se desarrolla o no debemos ultrapasar los criterios utilizados en el análisis de sus índices de ingreso per cápita que, estadísticamente mecanicistas, no alcanzan siquiera a expresar la verdad, y evitar, asimismo, los que se centran únicamente en el estudio de la renta bruta" (Freire, 1990a: 172). Y agrega en Pedagogía de la Indignación: "me parece una contradicción lamentable hacer un discurso progresista, revolucionario, y sostener una práctica negadora de la vida: una práctica que contamine el aire, las aguas, los campos, que arrase los bosques, destruya los árboles y amenace los animales terrestres y las aves" (Freire, 2012: 170). Es decir, la protección de la naturaleza también está explícitamente presente en su obra. Autores como Romo Torres incluso relacionan ciertos aspectos freireanos con algunos principios de la ecopedagogía expuestos por el pedagogo brasileño Moacir Gadotti, en particular, la ternura para con nuestro planeta. En este sentido, Romo Torres (2003) destaca el amor y el diálogo con los demás y la preocupación por los/as otros/as en y con sus diferencias como valores necesarios y aplicables al cuidado del ambiente.

En cualquier caso, también debe reiterarse que el enfoque transformador exige una responsabilidad, de ser parte, de tolerar, actuar, convivir y construir de forma conjunta y genuina una mejor realidad para todas las personas. Este sería el objetivo clave de 
la educación en derechos humanos y en desarrollo sostenible, compartido por Freire: fomentar el cambio individual, el empoderamiento, la crítica y el impulso para transformar y ser libres.

La interacción entre la educación en derechos humanos y en desarrollo sostenible aparece de manera explícita en la Agenda 2030, donde el ODS 4.7 indica que los Estados deben garantizar la educación para el desarrollo sostenible y los derechos humanos. Algunos de los indicadores para monitorear su cumplimiento son los índices de paridad (mujeres/hombres, zonas rurales/urbanas, la situación en materia de discapacidad y pueblos indígenas, por ejemplo) y el grado en que la educación para la ciudadanía global y la educación para un desarrollo sostenible (incluyendo igualdad de género y derechos humanos) se incorporan en las políticas nacionales de educación, los planes de estudio, la formación de los docentes y las evaluaciones de estudiantes.

Como se destacó anteriormente, recibir educación en derechos humanos y en desarrollo sostenible también es un derecho, que se desprende de tratados como el Pacto Internacional de Derechos Económicos, Sociales y Culturales, la Convención sobre los Derechos del Niño y el Protocolo de San Salvador, entre otros. Es decir, no se trata simplemente de algo noble, ético o moral: es jurídicamente obligatorio. Y es importante que los gobiernos entiendan este punto, pero también la sociedad, para estar en un mejor lugar de exigir lo que le corresponde. Por eso, es fundamental que se incorporen estos temas al currículum de las escuelas y las universidades, y que se brinden las herramientas necesarias al cuerpo docente para poder enseñar apropiadamente estas cuestiones.

A pesar de que actualmente está fuera de discusión que los Estados están obligados a garantizar el derecho a la educación y que deben introducir la educación en derechos humanos y en desarrollo sostenible en todos los niveles, lamentablemente esto no quiere decir que se cumpla (IIDH, 2007). Por ejemplo, solamente un tercio de los países destinan entre el $15 \%$ y el $20 \%$ de su presupuesto a educación, tal como recomienda el Marco de Acción Educación 2030 (Consejo Económico y Social, 2019). Asimismo, solo la mitad de los Estados miembros de Unesco reportaron haber integrado la educación en derecho sostenible en políticas públicas relevantes (Unesco, 2015). Además, Unesco (2019) también advirtió que el monitoreo del ODS 4.7 todavía permanece como desafío porque aún no se adoptó una metodología para trabajar con los indicadores y reclamó que para monitorear esta meta adecuadamente se necesitan 280 millones de dólares por año cuando, actualmente, se destinan 148 millones. El organismo concluyó categóricamente que "el mundo aún está lejos de poder cumplir los compromisos internacionales de educación” (Unesco, 2019: 12).

Este punto no debe desmotivar el activismo sino que, por el contrario, evidencia la necesidad de seguir luchando. Un mundo más justo solo es posible a través del respeto y la garantía de los derechos humanos y del ambiente. No se puede pensar una emancipación o una liberación sin estos elementos. La falta de cumplimiento de estos objetivos no significa que la educación sobre estos temas no sea un paso elemental para lograrlos. Seguramente, también habrá otras formas, métodos y herramientas, que deberían ser complementarias y adicionales.

\section{A modo de cierre}

Paulo Freire impulsó "desde sus propias prácticas y reflexiones la defensa y la promoción de los derechos humanos" (El Achkar, 2002: 115). Al mismo tiempo, la educación en derechos humanos y desarrollo sostenible es una de las mejores herramientas para 
alcanzar los objetivos planteados por el pedagogo brasileño a lo largo de su vida. Es un recurso fundamental para garantizar la dignidad de las personas y prevenir crímenes y atrocidades, que "pretende avanzar en la convivencia y en el progreso humano, teniendo en cuenta aspectos como el cambio climático, la reducción de la pobreza, el consumo sostenible, la paz y la seguridad ciudadana" (Acevedo Suárez y Báez Pimiento, 2018: 71). Por eso, es necesario incorporarla efectivamente en la formación de todo individuo, a través de todos los canales posibles.

Existen otras corrientes con objetivos similares, llamadas educación por la ciudadanía, educación para la paz, formación en valores, educación ética o educación para la convivencia, la tolerancia y no la discriminación. Aquí se utilizó el término educación en derechos humanos para referir a las actividades que tengan el objetivo de promover el respeto de los derechos humanos, contribuir a la prevención de abusos y promocionar una cultura de derechos humanos. No obstante, como cuestionan autores como Magendzo y Toledo-Jofré (2015), a pesar de que todos estos programas apunten a construir una sociedad más justa e igualitaria, es cierto que aún no está claro cómo conviven; de hecho, a veces se generan confusiones y tanta diversidad de nombres puede provocar incluso cierto rechazo frente a toda la corriente en general.

Futuras investigaciones podrán evaluar esta convergencia y proponer una solución superadora, que podría ser incluir todos los programas bajo un solo nombre ya existente, o inventar un nuevo término que englobe a todos los conceptos. Es importante unir esfuerzos y no generar divisiones cuando los propósitos son tan coincidentes. Asimismo, también será elemental diferenciar entre enseñar derechos humanos y bajar línea, promover la flexibilidad y la tolerancia, no imponer puntos de vista propios, generar un pensamiento crítico genuino e incluir un abanico de temas que no se limite a contenidos particulares. Básicamente, se trata de volver a la idea original de los derechos humanos, cuando surgieron formalmente con la Declaración Americana de Derechos y Deberes del Hombre y la Declaración Universal de Derechos Humanos en 1948, y tratar de escaparle a la manipulación que sufrió el término en los años posteriores, ya sea por cuestiones de política internacional, como la Guerra Fría, o de política nacional argentina, como las dictaduras, el peronismo, el radicalismo, el kirchnerismo y el macrismo.

La educación en derechos humanos y desarrollo sostenible debería trascender más allá de políticas partidarias, no solo porque se trata de valores con un altísimo consenso universal, sino porque además es un derecho que el Estado argentino voluntariamente se comprometió a garantizar mediante la firma de acuerdos internacionales y también a través de su normativa local. Sin embargo, si los derechos humanos son solo una teoría interesante pero que no transforma las vidas, la manera de pensar, la forma de sentir o cómo se relacionan las personas unas con otras, entonces no es más que un discurso vacío sin consecuencias prácticas (Mujica, 2002). Como enseña Mónica Pinto (2010: 6): "comprender los derechos humanos es entender que están presentes en el aquí y ahora, desde los contextos cercanos hasta los distantes: en las situaciones de la vida personal como de la comunidad local, en los problemas del país como los de la región y el mundo. Es reconocer que en su defensa y promoción se juega la vida y la felicidad de las personas y de los pueblos".

Volviendo a la conexión con Freire y su espíritu crítico, emancipador y liberador, el potencial de empoderamiento "es una de las principales distinciones que busca tener la educación en derechos humanos" (Tibbitts y Kirchschläger, 2010: 29). Más aún, también busca "vincular la conciencia crítica con la acción social para superar estructuras sociales opresivas" (Magendzo y Pavez, 2018: 144). Nuevamente, es evidente la influencia del pedagogo brasileño y la coincidencia entre los propósitos. En este sentido, lo que deben buscar quienes enseñan derechos humanos es "contribuir a 
que cada persona conquiste su derecho a existir y desarrolle su capacidad para crear condiciones donde los derechos humanos sean una realidad vigente" (Mujica, 2002: 3). Para ello, es necesario, por un lado, implementar un enfoque transversal que permita mirar e interpretar la realidad desde la óptica de los derechos humanos (Rodino, 2014) y no tenerle miedo al diálogo, la controversia y el debate: "los ciudadanos no ponen en riesgo a la sociedad cuando piensan y participan críticamente, sino cuando aceptan dócilmente las seducciones del poder de turno, cuando admiten la realidad tal como se les presenta y cuando renuncian a buscar alternativas" (Siede, 2010: 37).

Finalmente, también es necesario advertir que la educación en derechos humanos y desarrollo sostenible no podrá mejorar el mundo por sí sola. Es decir, para hacer realidad estos objetivos es fundamental un cambio estructural y cultural, que si bien puede estar influido por todos los niveles del sistema educativo formal, también se construye según los medios de comunicación, las familias, religiones, tradiciones, amistades, etcétera. Entonces, la educación formal podrá ser una herramienta importante para mejorar el mundo, pero no necesariamente será siempre la principal, la única o la más efectiva, al igual que puede suceder con la política, el derecho, la economía o hasta los medios de comunicación y las redes sociales. En resumen, el potencial que puede tener la educación no debe generar expectativas desmedidas y será necesario trabajar en conjunto con otras disciplinas.

\section{Conclusión}

El propósito de este ensayo de reflexión teórica fue, en primer lugar, aprovechar el centenario del nacimiento de Paulo Freire para vincular algunos puntos y valores principales de su obra con los objetivos que persigue actualmente la educación en derechos humanos. En segundo lugar, se propuso incluirla en la conexión con la educación en desarrollo sostenible, imprescindible para alcanzar estas metas. En tercer lugar, es importante colaborar en la difusión de estas ideas, valores y programas educativos en cada canal existente. Desde ya, no alcanza solo un ensayo para analizar toda la complejidad del pensamiento y la obra de Freire y vincularla con la educación en derechos humanos y desarrollo sostenible, pero sí al menos para plantear algunas ideas.

En este sentido, se buscó hacer un aporte desde la óptica jurídica, ya que el derecho, la ley y las normas pueden servir de herramientas de apoyo para conseguir estos fines. Además, el enfoque interdisciplinario es clave para alcanzar el efectivo cumplimiento de los derechos humanos. Ni la educación ni el derecho podrán por sí solos conseguir los cambios buscados. Como sostiene Pérez Aguirre (2007: 49), "estamos mal educados para los derechos humanos". Será responsabilidad de todas las personas aportar para revertir esto, siempre desde la sensibilidad y el interés por ayudar a las personas más necesitadas, como muestra el legado de Freire. 


\section{Bibliografía}

" Abril, D. (2003). Pensar globalmente, actuar localmente... ¡Desde el aula! En Gadotti, M.; Gomez, M. y Freire, L. (eds.). Lecciones de Paulo Freire cruzando fronteras: experiencias que se completan. Buenos Aires, Consejo Latinoamericano de Ciencias Sociales/ CLACSO

" Acevedo Suárez, A. y Báez Pimiento, A. (2018). La educación en cultura de paz. Herramienta de construcción de paz en el posconflicto. Revista Reflexión Política, 20(40).

"Asamblea General de las Naciones Unidas. (2011). Declaración de las Naciones Unidas sobre educación y formación en materia de derechos humanos. A/RES/66/137. Nueva York, ONU.

"Asamblea General de las Naciones Unidas. (2018). Informe de la Relatora Especial sobre el derecho a la educación, A/73/262. Nueva York, ONU.

"Capalbo, L.; Ander Egg, E.; Elizalde Hevia, A.; Grinberg, M. y Laszlo, E. (2011). Decrecer con equidad: Nuevo paradigma civilizatorio. Buenos Aires, Ciccus.

"Cho, H. (2018). Enseñando la DUDH en el marco de una ciudadanía global: una reflexión personal. En Magendzo, A. y Morales, P. (eds.). Pedagogía y didáctica de la Declaración Universal de los Derechos Humanos a setenta años de su promulgación. Santiago de Chile, Ediciones Universidad Academia de Humanismo Cristiano.

"Comisión Interamericana de Derechos Humanos (CIDH). (2019). Informe Empresas y Derechos Humanos: Estándares Interamericanos. Estados Unidos.

"Comité de los Derechos del Niño. (2016). Observación general núm. 20 (2016) sobre la efectividad de los derechos del niño durante la adolescencia, CRC/C/GC/2o. Ginebra, ONU.

"Comité para la Eliminación de la Discriminación contra la Mujer. (2017). Recomendación general núm. 36 (2017) sobre el derecho de las niñas y las mujeres a la educación. Ginebra. CEDAW/C/GC/36, ONU.

»Consejo Económico y Social de Naciones Unidas. (2019). Edición especial: progresos realizados para lograr los Objetivos de Desarrollo Sostenible, E/2019/68. Ginebra, ONU.

"Corte Interamericana de Derechos Humanos (Corte IDH). (2017). Medio ambiente y derechos humanos (obligaciones estatales en relación con el medio ambiente en el marco de la protección y garantía de los derechos a la vida y a la integridad personalinterpretación y alcance de los artículos 4.1 y 5.1, en relación con los artículos 1.1 y 2 de la Convención Americana sobre Derechos Humanos). Opinión Consultiva OC23/17. San José de Costa Rica.

"Corte Interamericana de Derechos Humanos (2020). Caso Comunidades Indígenas Miembros de la Asociación Lhaka Honhat (Nuestra Tierra) vs. Argentina. San José de Costa Rica.

"Cussianovich, A. (2010). Aprender la condición humana: ensayo sobre pedagogía de la ternura. Lima, Ifejant.

"El Achkar, S. (2002). Una mirada a la Educación en Derechos Humanos desde el pensamiento de Paulo Freire. En Mato, D. (coord.). Estudios y otras Prácticas Intelectuales Latinoamericanas en Cultura y Poder. Caracas, Consejo Latinoamericano de Ciencias Sociales (CLACSO), CEAP, FACES y Universidad Central de Venezuela. 
» Fernández, M. (2019). Repensando la enseñanza y el aprendizaje de los derechos humanos: una perspectiva pedagógica descolonizadora. En Ramírez, R. y Pisarello, M. (comps.). Educación Superior y Derechos Humanos: Política, prácticas y dispositivos a 100 años de la Reforma Universitaria. Universidad Autónoma de Entre Ríos.

» Fisas, V. (1998). Cultura de paz y gestión de conflictos. Barcelona, Icaria/Unesco.

» Franco Sentis, E.; Luque, D.; Muñoz, P. y Silva, C. (2018). Formando educadoras/ es garantes de derechos humanos: reflexiones, aprendizajes y desafíos desde la educación universitaria. En Magendzo, A. y Morales, P. (eds.). Pedagogía y didáctica de la Declaración Universal de los Derechos Humanos a setenta años de su promulgación. Santiago de Chile, Ediciones Universidad Academia de Humanismo Cristiano.

" Freire, P. (1999a). Pedagogía del Oprimido. México, Siglo XXI.

"Freire, P. (1999b). Pedagogía de la autonomía: Saberes necesarios para la práctica educativa. México, Siglo XXI.

"Freire, P. (2002). Pedagogía de la esperanza: un reencuentro con la Pedagogía del Oprimido. México, Siglo XXI.

》Freire, P. (2012). Pedagogía de la indignación. Buenos Aires, Siglo XXI.

"Instituto Danés de Derechos Humanos. (2013). Recursos para la educación en Derechos Humanos. Dinamarca. Disponible en https://www.humanrights.dk/files/ media/dokumenter/udgivelser/hre_esp.pdf

»Instituto Interamericano de Derechos Humanos (IIDH). (2007). VI Informe Interamericano de la Educación en Derechos Humanos. San José de Costa Rica. Disponible en https://www.iidh.ed.cr/multic/UserFiles/Biblioteca/ IIDH/3_2010/484ees6a-c4a5-4c7b-a29b-62f9aeze651a.pdf

»Instituto Interamericano de Derechos Humanos (2013). El Derecho a la Educación en Derechos Humanos en las Américas. San José de Costa Rica. Disponible en https:// www.iidh.ed.cr/IIDH/media/1517/informe-interamericano-el-derecho-a-la-edh-2013. pdf.

"Kohan, W. (2020). Paulo Freire más que nunca: una biografía filosófica. Buenos Aires, Consejo Latinoamericano de Ciencias Sociales-CLACSO.

»Magendzo, A. (2001). La pedagogía de los derechos humanos. Lima, Material publicado por el IPEDEHP. Disponible en https://docplayer.es/13643923-La-pedagogia-de-losderechos-humanos.html.

» Magendzo, A. y Pavez, J. (2018). Educando en la declaración universal de los derechos humanos desde una mirada controversial. En Magendzo, A. y Morales, P. (eds.). Pedagogía y didáctica de la Declaración Universal de los Derechos Humanos a setenta años de su promulgación. Santiago de Chile, Ediciones Universidad Academia de Humanismo Cristiano.

» Magendzo, A. y Toledo-Jofré, M. (2015). Educación en derechos humanos: Estrategia pedagógica-didáctica centrada en la controversia. Revista Electrónica Educare, 19(3).

» Medici, A. (2018). Articulación de docencia-investigación-extensión para una práctica pedagógica crítica en la enseñanza de los Derechos Humanos. Revista Derechos en Acción, 7(7).

»Mujica, R. M. (2002). La metodología de la educación en derechos humanos. Revista Interamericana de Derechos Humanos, 36. Disponible en http://www.corteidh.or.cr/ tablas/Ro6835-13.pdf.

"Pérez Aguirre, L. (2007). Si digo educar para los derechos humanos. Revista DEHUIDELA, 7(15). 
"Pinto, M. (2010). La enseñanza de Derechos Humanos en la Universidad de Buenos Aires. Academia: Revista sobre enseñanza del Derecho, 8(16). Disponible en www.derecho.uba.ar/publicaciones/rev_academia/revistas/16/la-ensenanza-dederechos-humanos-en-la-universidad-de-buenos-aires.pdf.

»Requesens Galnares, A. (2006). Retos a la educación en derechos humanos. En Educación en Derechos Humanos, Programa de Cooperación sobre Derechos Humanos México-Comisión Europea. Disponible en http://ru.juridicas.unam.mx/ xmlui/handle/123456789/28222.

» Rodino, A. (2014). La institucionalización de la educación en derechos humanos en América Latina: avances, desafíos y una propuesta de prioridades. Sociedade e Cultura (16).

"Romo Torres, R. (2003). Contribuciones freirianas al pensamiento latinoamericano. En Gadotti, M.; Gómez, M. y Freire, L. (eds.). Lecciones de Paulo Freire cruzando fronteras: experiencias que se completan. Buenos Aires, Consejo Latinoamericano de Ciencias Sociales-CLACSO.

» Ronconi, L. (2017). La enseñanza en derechos humanos en las Facultades de Derecho en Argentina: desafíos pendientes. Revista Pedagogía Universitaria y Didáctica del Derecho, 4(1).

»Siede, I. (2010). Ciencias Sociales en la escuela: sentidos de la enseñanza. En Siede, I. (ed.). Ciencias Sociales en la escuela: criteios y propuestas para la enseñanza. Buenos Aires, Aique.

» Sikkink, K. (2017). Evidence for hope: making human rights work in the 21st century. Princeton University Press.

» Tibbitts, F. (2017). Evolution of Human Rights Education Models. En Bajaj, M. (ed.). Human Rights Education: Theory, Research, Praxis. Filadelfia, University of Pennsylvania Press.

» Tibbitts, F. y Fernekes, W. (2011). Human Rights Education. En Totten, S. y Pederson, J. E. (eds.). Teaching and Studying Social Issues: Major Programs and Approaches. Charlotte, Information Age Publishing Inc.

» Tibbitts, F. y Kirchschläger, P. G. (2010). Perspectives of Research on Human Rights Education. Journal of Human Rights Education, 2(1).

» Tibbitts, T. y Nelson, R. (2018). El tratamiento de los seres humanos como objetos: cómo los estudiantes norteamericanos ven los derechos humanos y cómo aprendieron sobre ellos en la escuela. En Magendzo, A. y Morales, P. (eds.). Pedagogía y didáctica de la Declaración Universal de los Derechos Humanos a setenta años de su promulgación. Santiago de Chile, Ediciones Universidad Academia de Humanismo Cristiano.

" United Nations Educational, Scientific and Cultural Organization (Unesco); United Nations Development Program (UNDP), United Nations Population Fund (UNFPA), the Office of the United Nations High Commissioner for Refugees (UNHCR), United Nations Children's Fund (UNICEF), UN Women, World Bank Group, International Labour Organization (ILO). (2015). Education 2030: Incheon Declaration and Framework for action for the implementation of Sustainable Development Goal 4. Ensure inclusive and equitable quality education and promote lifelong learning opportunities for all. Disponible en https://unesdoc.unesco.org/ark:/48223/pfoooo245656

»United Nations Educational, Scientific and Cultural Organization (Unesco); United Nations Development Program (UNDP), United Nations Population Fund (UNFPA), the Office of the United Nations High Commissioner for Refugees (UNHCR), United Nations Children's Fund (UNICEF), UN Women, World Bank Group, International 
Labour Organization (ILO) (2016). Action for Climate Empowerment: Guidelines for accelerating solutions through education, training and public awareness. Disponible en https://unesdoc.unesco.org/ark:/48223/pfoooo246435

»United Nations Educational, Scientific and Cultural Organization (Unesco); United Nations Development Program (UNDP), United Nations Population Fund (UNFPA), the Office of the United Nations High Commissioner for Refugees (UNHCR), United Nations Children's Fund (UNICEF), UN Women, World Bank Group, International Labour Organization (ILO) (2019). Cumplir los compromisos: ¿van los países por el buen camino para alcanzar el ODS 4? Disponible en https://unesdoc.unesco.org/ ark:/48223/pfoooo369009_spa

\section{Alan Vogelfanger}

Abogado, Universidad de Buenos Aires. Magíster en Estudios Legales Internacionales, con especialización en Derechos Humanos, American University, Washington College of Law. Docente de "Derechos Humanos y Garantías" y "Derecho Internacional de los Derechos Humanos", Facultad de Derecho, Universidad de Buenos Aires. avogelfanger@gmail.com 